\title{
Genetic testing for atrial septal defect
}

\author{
Yeltay Rakhmanov ${ }^{1}$, Paolo Enrico Maltese ${ }^{1 \star}$, Alessandra Zulian ${ }^{2}$, Tommaso Beccari ${ }^{3}$, \\ Munis Dundar ${ }^{4}$ and Matteo Bertelli ${ }^{1,2}$
}

\begin{abstract}
Atrial septal defect (ASD) is a congenital heart defect characterized by an opening in the atrial septum. About $1 / 3$ of patients with Noonan syndrome caused by mutation in the PTPN11 gene have ASD. The prevalence of ASD is estimated at 100 per 100,000 live births. ASD may have autosomal dominant or recessive inheritance. This Utility Gene Test was prepared on the basis of an analysis of the literature and existing diagnostic protocols. It is useful for confirming diagnosis, as well as for differential diagnosis, couple risk assessment and access to clinical trials.
\end{abstract}

Keywords: Atrial septal defect, ostium primum, heart murmur, EBTNA UTILITY GENE TEST

'MAGI's Lab, Rovereto, Italy

${ }^{2}$ MAGI Euregio, Bolzano, Italy

${ }^{3}$ Department of Pharmaceutical Sciences, University of Perugia, Perugia, Italy

${ }^{4}$ Department of Medical Genetics, Erciyes University Medical School, Kayseri, Turkey

*Corresponding author: P. E. Maltese E-mail: paolo.maltese@assomagi.org

DOI: 10.2478/ebtj-2018-0035
(C) 2018 Authors. This work was licensed under the Creative Commons AttributionNonCommercial-NoDerivs 3.0 License.

\section{Atrial septal defect}

(Other synonyms: Atrial septal defect, primum type, atrial septal defect, secundum type)

\section{General information about the disease}

Atrial septal defect (ASD) is the most common congenital heart defect and is characterized by an aperture in the atrial septum. It is estimated to be a major cause of prenatal birth losses. ASD has different morphological types determined by the location of the defect. The defect usually borders the oval fossa and its antero-inferior rim. Communication between the two atria, such as superior or inferior sinus venosus defects, coronary sinus defect and ostium primum defect, are very rarely observed (1). Variations in several genes explain the mechanism of defect development. Sznajer et al. reported that $38.25 \%$ patients with Noonan Syndrome caused by PTPN11 variation had heart pathologies and $26.90 \%$ of this cohort developed ASD (2).

Patients with ASD remain asymptomatic throughout most of their childhood. Most patients consult their physician for a heart murmur. Infants with ASD may have tachypnoea, slow weight gain and recurrent respiratory infections (3, 4). Large size ASD might manifest with different clinical symptoms such as didyspnea, fatigue, exercise intolerance, or palpitations (5). Right-sided heart failure may be accompanied by cyanosis, syncope and peripheral edema (6).

The prevalence of ASD is estimated at 100 per 100,000 live births (7). About $65-70 \%$ of patients with a secundum defect, roughly $50 \%$ of those with a primum atrial septal defect, and $40-50 \%$ of those with a sinus venosus defect are females (5).

To visualize ASD and characterize its size, determine the direction of flow, evaluate associated abnormalities, examine the right heart and estimate pulmonary/systemic flow ratio (6), the methods used include echocardiogram, electrocardiogram, chest radiogram, spinecho MR imaging, CT and diagnostic catheterisation. To diagnose inheritance pattern, genetic testing is used. Differential diagnosis should consider ASD caused by Down syndrome and other chromosomal disorders. 


\section{Autosomal dominant non-syndromic ASD}

- ASD1 (OMIM disease 108800) caused by variations in a locus mapping to chromosome $5 \mathrm{p}$;

- ASD2 (OMIM disease 607941) - GATA4 (OMIM gene 600576);

- ASD3 (OMIM disease 614089) - MYH6 (OMIM gene 160710);

- ASD4 (OMIM disease 611363) - TBX20 (OMIM gene 606061);

- ASD5 (OMIM disease 612794) - ACTC1 (OMIM gene 102540);

- ASD6 (OMIM disease 613087) - TLL1 (OMIM gene 606742);

- ASD7, with or without AV conduction defects (OMIM disease 108900) - NKX2-5 (OMIM gene 600584);

- ASD8 (OMIM disease 614433) - CITED2 (OMIM gene 602937);

- ASD9 (OMIM disease 614475) - GATA6 (OMIM gene 601656);

\section{Autosomal dominant syndromic ASD}

- Noonan Syndrome 1 (NS1, OMIM disease 163950) PTPN11 (OMIM gene 176876);

- Holt-Oram syndrome (HOS, OMIM disease 142900) TBX5 (OMIM gene 601620);

- Cardiofaciocutaneous syndrome 1 (CFC1, OMIM disease 115150) - BRAF (OMIM gene 164757);

- Mowat-Wilson syndrome (MOWS, OMIM disease 235730) - ZEB2 (OMIM gene 605802).

- Floating-Harbor syndrome (FLHS, OMIM disease 136140) - SRCAP (OMIM gene 611421)

\section{Autosomal recessive ASD}

- Ellis-van Creveld syndrome (EVC, OMIM disease 225500) - EVC (OMIM gene 604831) or EVC2 (OMIM gene 607261).

Pathogenic variants may include missense, nonsense, splicing, small insertions, small deletions and small indels.

\section{Aims of the test}

- To determine the gene defect responsible for the disease;

- To confirm clinical diagnosis;

- To assess the recurrence risk and perform genetic counselling for at-risk/affected individuals.

\section{Test characteristics}

\section{Specialist centers/ Published Guidelines}

The test is listed in the Orphanet database and is offered by 16 accredited medical genetic laboratories in the EU, and in the GTR database, offered by 10 accredited medical genetic laboratories in the US.

Guidelines for clinical use of the test are described in Genetics Home Reference (ghr.nlm.nih.gov).

\section{Test strategy}

Clinically distinguishable syndromes can be analyzed by sequencing only those genes known to be associated with that specific disease using Sanger or Next Generation Sequencing (NGS); if the results are negative, or more generally if clinical signs are ambiguous for diagnosis, a multi-gene NGS panel is used to detect nucleotide variations in coding exons and flanking introns of the above genes.

Potentially causative variants and region with low coverage are Sanger-sequenced. Sanger sequencing is also used for family segregation studies.

Multiplex Ligation Probe Amplification (MLPA) is used to detect duplications and deletions in TBX5 gene.

To perform molecular diagnosis, a single sample of biological material is normally sufficient. This may be $1 \mathrm{ml}$ peripheral blood in a sterile tube with $0.5 \mathrm{ml} \mathrm{K}$ EDTA or $1 \mathrm{ml}$ saliva in a sterile tube with $0.5 \mathrm{ml}$ ethanol $95 \%$. Sampling rarely has to be repeated.

Gene-disease associations and the interpretation of genetic variants are rapidly developing fields. It is therefore possible that the genes mentioned in this note may change as new scientific data is acquired. It is also possible that genetic variants today defined as of "unknown or uncertain significance" may acquire clinical importance.

\section{Genetic test results}

\section{Positive}

Identification of pathogenic variants in the above genes confirms the clinical diagnosis and is an indication for family studies.

A pathogenic variant is known to be causative for a given genetic disorder based on previous reports, or predicted to be causative based on loss of protein function or expected significant damage to proteins or protein/protein interactions. In this way it is possible to obtain a molecular diagnosis in new/other subjects, establish the risk of recurrence in family members and plan preventive and/or therapeutic measures.

\section{Inconclusive}

Detection of a variant of unknown or uncertain significance (VUS): a new variation without any evident pathogenic significance or a known variation with insufficient evidence (or with conflicting evidence) to indicate it is likely benign or likely pathogenic for a given genetic disorder. In these cases, it is advisable to extend testing to the patient's relatives to assess variant segregation and clarify its contribution. In some cases, it could be necessary to perform further examinations/tests or to do a clinical reassessment of pathological signs.

\section{Negative}

The absence of variations in the genomic regions investigated does not exclude a clinical diagnosis but suggests the possibility of:

- alterations that cannot be identified by sequencing, such as large rearrangements that cause loss (deletion) or gain (duplication) of extended gene fragments; 
- sequence variations in gene regions not investigated by this test, such as regulatory regions (5' and 3' UTR) and deep intronic regions;

- variations in other genes not investigated by the present test.

\section{Unexpected}

Unexpected results may emerge from the test, for example information regarding consanguinity, absence of family correlation or other genetically-based diseases.

\section{Risk for progeny}

If the identified pathogenic variant has autosomal dominant transmission, the probability that an affected carrier transmit the disease variant to his/her children is $50 \%$ in any pregnancy, irrespective of the sex of the child conceived.

In autosomal recessive mutations, both parents are usually healthy carriers. In this case, the probability of transmitting the disorder to the offspring is $25 \%$ in any pregnancy of the couple, irrespective of the sex of the child. An affected individual generates healthy carrier sons and daughters in all cases, except in pregnancies with a healthy carrier partner. In these cases, the risk of an affected son or daughter is $50 \%$.

\section{Limits of the test}

The test is limited by current scientific knowledge regarding the gene and disease.

Analytical sensitivity (proportion of positive tests when the genotype is truly present) and specificity (proportion of negative tests when the genotype is not present)

NGS Analytical sensitivity $>99.99 \%$, with a minimum coverage of 10X; Analytical specificity 99.99\%.

SANGER Analytical sensitivity >99.99\%; Analytical specificity 99.99\%.

\section{Clinical sensitivity (proportion of positive tests} if the disease is present) and clinical specificity (proportion of negative tests if the disease is not present)

Clinical sensitivity: the variations in the aforementioned genes are linked to ASD, but may be individual variations (identified in one or few families) and total epidemiological data is therefore not available.
Clinical sensitivity will be estimated based on internal cases. Clinical specificity is estimated at approximately $99 \%$ (8).

\section{Prescription appropriateness}

The genetic test is appropriate when:

a) the patient meets the diagnostic criteria for ASD;

b) the sensitivity of the test is greater than or equal to that of tests described in the literature.

\section{Clinical utility}

\section{Clinical management}

Utility

Confirmation of clinical diagnosis

Yes

Differential diagnosis Yes

Couple risk assessment Yes

Availability of clinical trials can be checked on-line at https://clinicaltrials.gov/

\section{References}

1. Beridze N, Frishman WH. Vascular Ehlers-Danlos syndrome: pathophysiology, diagnosis, and prevention and treatment of its complications. Cardiol Rev 2012; 20(1): 4-7.

2. Sznajer Y, Keren B, Baumann C, Pereira S, Alberti C, Elion J, Cavé H, Verloes A. The spectrum of cardiac anomalies in Noonan syndrome as a result of mutations in the PTPN11 gene. Pediatrics 2007; 119(6): e1325-31.

3. Andrews R, Tulloh R, Magee A, Anderson D. Atrial septal defect with failure to thrive in infancy: hidden pulmonary vascular disease? Pediatr Cardiol 2002; 23(5): 528-530.

4. Lammers A, Hager A, Eicken A, Lange R, Hauser M, Hess J. Need for closure of secundum atrial septal defect in infancy. J Thorac Cardiovasc Surg 2005; 129(6): 1353-57.

5. Geva T, Martins JD, Wald RM. Atrial septal defects. Lancet 2014; 383(9932): 1921-32.

6. Martin SS, Shapiro EP, Mukherjee M. Atrial septal defects - clinical manifestations, echo assessment, and intervention. Clin Med Insights Cardiol 2015; 23;8(Suppl 1): 93-8.

7. Botto LD, Correa A, Erickson JD. Racial and temporal variations in the prevalence of heart defects. Pediatrics 2001; 107(3): E32.

8. Chen B, Gagnon M, Shahangian S, Anderson NL, Howerton DA, Boone JD; Centers for Disease Control and Prevention (CDC). Good Laboratory Practices for Molecular Genetic Testing for Heritable Diseases and Conditions. MMWR Recomm Rep. 2009; 58(RR6): 1-37; quiz CE-1-4. 